ИЗВЕСТИЯ АКАДЕМИИ НАУК ЭСТОНСКОИ ССР. ТОМ ХІІІ СЕРИЯ ФИЗИКО-МАТЕМАТИЧЕСКИХ И ТЕХНИЧЕСКИХ НАУК. 1964, № 4

\title{
ОДНО КОДИРОВАНИЕ ДЛЯ МОДЕЛИРОВАНИЯ ЦИФРОВЫХ СХЕМ
}

\section{Х. САЛУМ}

Произвольная композиция автоматов (в смысле, данном В. М. Глушковым [']) может быть охарактеризсвана

1) операторами отдельных автоматов

2) входными связями (входными векторами) этих автоматов

3) выходными связями (выходными векторами) этих автоматов

и промоделирована на МЦВМ. Считая для каждого конкретного случая кодирование состояний, входов и выходов уже выбранным, можно составить формальное кодирование для описания произвольной структурной схемы. Кодирование упрощается, если выделить некоторое количество элементов памяти, управляемых программой, для моделирования работы схемы.

Основными составными частями кодирования являются: грамм,

1) операторы отдельных автоматов, записанные в виде подпро-

2) массивы данных о состояниях, параметрах и связях в схеме.

В данной работе предлагается предварительный вариант кодирования для моделирования на МЦВМ произвольных цифровых схем. Исходные данные о схеме должны быть представлены в виде логической схемы на уровне логических элементов и регистров.

Моделирование работы структурной схемы производится по моментам автоматного времени.

Основными символами синтаксической записи прнменяемых понятий являются символы, использованные для описания АЛГОЛ-60 в [2]. K этому списку добавлен символ для обозначения адреса второго ранга [3]. Часть обозначений, особенно в разделах 1 и 2 , еще не полностью определилась и дана описательно.

Для упрощения кодирования часть элементов памяти выделена и управляется моделирующей программой. Время моделирования одного такта работы АУ ЦВМ, подобной М-3, равно 6-10 сек.

Работа выполнена в Институте кибернетики Академии наук Украинской ССР под руководством академика АН СССР доктора физико-математических наук В. М. Глушкова.

\section{1. Элементы}

Қаждому логическому элементу в подлежащей моделированию схеме сопоставляется кодирующее понятие - элемент. Кодовый элемент характеризуется логическим содержанием и обозначается соответствую- 
щей этому содержанию большой латинской буквой. Элементы с одинаковым логическим содержанием - для краткости называемым дальше типом элемента - объединяются в двухразмерный массив, и каждому отдельному элементу присваиваются 2 индекса. Әлементы одного типа с одинаковым первым индексом составляют регистр.

Элемент типа «активизированньй» по своему существу является промежуточным между другими элементами и регистрами. Его назначение - принудительно управлять другими элементами.

При моделировании программа сопоставляет каждому элементу оператор, информационно моделирующий работу описываемого элемента схемы. Соответствие между элементами и операторами дано в табл. 1. Для моделирования работы оператор шины не нужен, так как шины выполняют только функцию связи между другими элементами.

Таблица 1

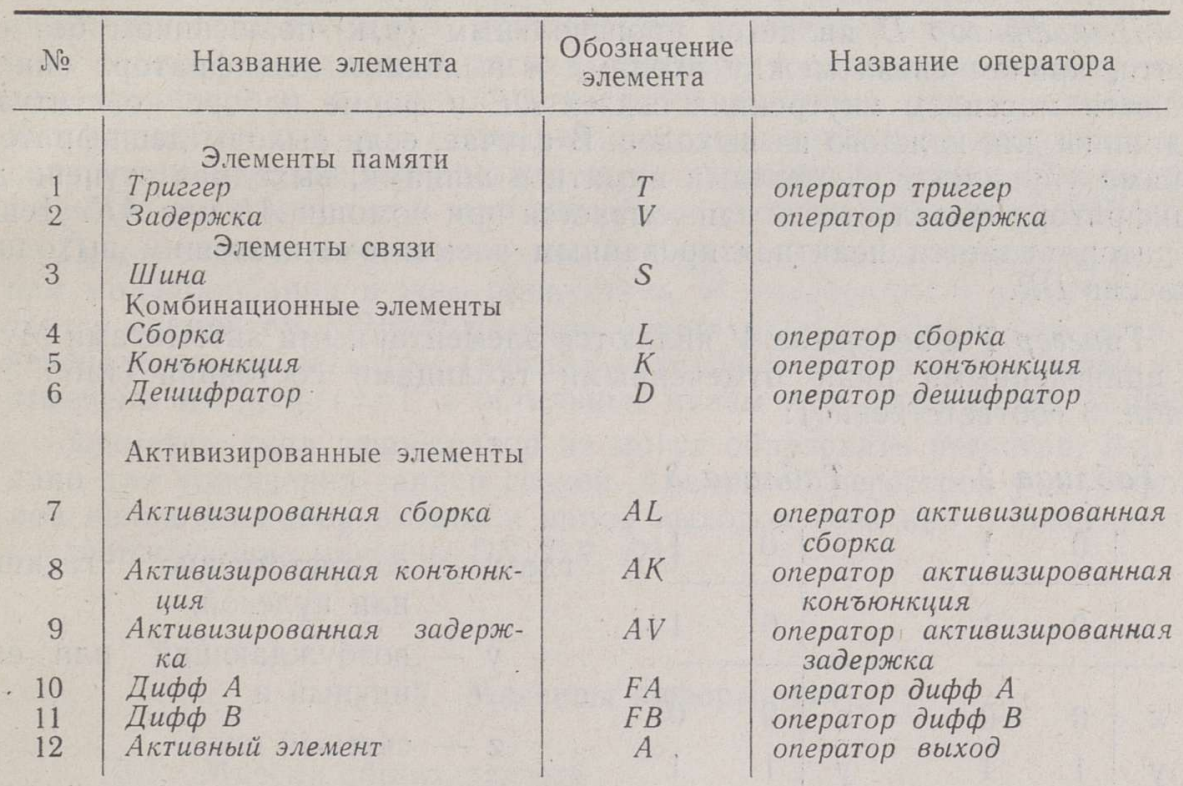

Входящий в состав списка элементов активный элемент необходим в основном для моделирования внешних управляющих сигналов и формирователей.

Получаемая при записи модель схємы не содержит в явном виде обозначений отдельных элементов. Қодовый элемент является понятием, объединяющим в одно целое соответствующие ему массивы. Индексы, соответствующие каждому элементу, указывают связанные с ним элементы массивов.

В предлагаемом кодировании для записи цифровых схем используются следующие массивы:

1. Входных связей $R S$

2. Выходных связей $R R$

3. Внутренних связей $R E$

4. Состояний $R 1$ и $R 2$.

Все эти массивы подробно описаны в разделе 3. Первый символ идентификатора массива $-R-$ является обобщенным для краткости за- 
писи. При записи схемы в кодированном для моделирования виде $R$ заменяется соответствуюшим для типа элемента символом (K|L D $T|V| S|A K \backslash A L| A V|F A| F B \mid A)$.

Выбор набора элементов, использованного в данном предварительном варианте кодирования, сделан эмпирически, причем система является логически полной.

Любой из элементов является частным случаем полного абстрактного автомата (Мили или Мура).

Комбинационный элемент является элементом без памяти (тривиальный случай автоматов Мили).

Сигнал на выходе (двухвходовой) конъюнкц̧ии $K$ равен конъюнкцин сигналов, поданных на входы, а сигнал на выходе (двухвходовой) сборки $L$ равен дизъюнкции входных сигналов. Кратной записью двухвходовой сборки и двухвходовой конъюнкции можно описать сборки или конъюнкции с произвольным числом входов.

Дешифратор $D$ является произвольным (p, к)-полюсником без памяти, причем связи между входами і выходами дешифратора описываются массивом внутренних связей $D E$ в форме набора конституэнт единицы для каждого из выходов. В случае, если выходы дешифратора прямо управляют элементами памяти и шинами, выходная ступень депифратора выделяется и запысывается при помоци $A L$ или $A K$. Дешифратор является неактивизированным элементом, имеющим выходной массив $D R$.

Tриггер $T$ и задержка $V$ являются элементарными автоматами Мура с прнведенными ниже отмеченными таблицами состояний (табл. 2 и табл. 3 соответственно):

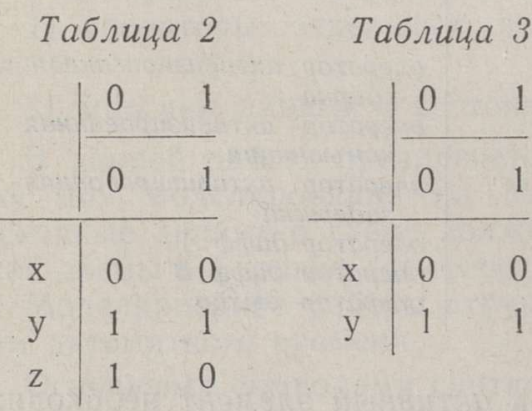
где $\mathrm{x}$ - соответственно гасяций или нулевой,
у - возбуждающий или еди- ничный и
z - счетный вход.

Шина $S$ является элементом связи между активизированными конъюнкциями. Шина возбуждается $(S 1::=1)$. на время одного цикла моделирования от выхода подсоединенной к ней активизированной конъюнкций (имеющей нагрузку <выход на шины >).

Дифференцирующие элементы дифф $A$ и дифф $B$ выдают сигнал для работы входящего в них активного элемента соответственно в случае перехода элемента памяти из состояния 0 в состояние 1 и наоборот.

\section{2. Регистры}

Регистром является набор элементов одного типа с одинаковыл первым индексом.

Идентификатор элемента нигде, кроме семантических примечаний, не используется самостоятельно, а только в качестве начального символа для обозначенйя соответствуюшего массива. Исходя из этого, для 
экономии идентификаторов, сделано допущение, что при записи схемьс идентификатору элемента присваивается численное значение, указываюшее количество регистров, состоящих из элементов данного типа.

В разделе 1 б́ло указано, что для краткости записи свойств, общих для всех элементов, вместо перечисления идентификаторов всех элементов можно писать $R$. $R$ являетсуя максимальным значением первого индекса массива. Регистры из элементов каждого типа нумеруются отдельно, по порядку, начиная с 1 по $R$ (т. е. по численному значению идентификатора типа элементов в регистре).

Длина регистра $i$ - число $n i$ - указывает количество объединенных в один регистр элементов. Число пi записывается в массиве общих данных элементов $R O[i, 1]$.

$\mathrm{B}$ принципе регистр образуется путем произвольного присвоения одинакового первого индекса элементам одного типа. Практически целесо2 образно объединять в регистры элементы с максимально подобными: внешними связями (напр., конъюнкции, на первый вход которых поступает общий управляющий сигнал, триггеры с одинаковым набором входов, соединенных с одними регистрами активных или активизированных элементов, и т. д.). Такие регистры называются однородными и мо-делирующий их оператор может быть упрощен. Признак однородности регистра записывается в массиве общих данных элементом $R O[i, 2]$.

При желании можно отметить и этим исключить регистры, которые: при моделировании нужно пропустить. Используемость регистра отме-чается элементом $R O[i, 3]$ массива обших данных. Предполагается, что неиспользуемые регистры (регистры, которые нужно пропустить) будут отмечены $R O[i, 3]::=1$, а остальные нулем или отсутствием признака.

Элементы типа дешифратор не могут образовать регистра. Это сделано для упрощения записи связей. Аналогией регистров у дешифраторов являются набор входов и набор выходов (так как с ними связаны соответствующие массивы $D S, D R, D 1)$.

\section{3. Массивы информации}

\section{1. Массив общих данных}

<массив общих данньх>:: = integer array $R O[1: R, 1: 3]$, причем:

а) для регистров $R \neq D$

$R O[i, 1]::=<$ лина регистра $i>$

$R O[i, 2]::=\langle$ однородность регистра $i>$

$R O[i, 3]::=<$ используемость регистра $i>$

б) для дешифраторов

DO $[i, 1]::=<$ число входов дешифратора $i>$

DO $[i, 2]::=<$ число выходов дешифратора $i>$

DO $[i, 3]::=<$ используемость дешифратора $i>$

3.2. Массив входных связей

$<$ массив входных связей> : : = array $R S[1: R, 1: R O[i, 1], 1: 2$ (мак(имально)]

причем: 
a) массивы $D S[i, j], V S[i, j]$ и $A V S[i, j]$ имеют размерность $[1: R$, $1: R O[i, 1]]$, и в ячейке $R S[i, j]$ может быть записано $R 1[k, m]$ или $R 1[k, m]$;

б) массивы $K S[i, j, 1], K S[i, j, 2], \quad L S[i, j, 1], \quad L S[i, j, 2], A K S[i, j, 1]$, $A K S[i, j, 2], A L S[i, j, 1], A L S[i, j, 2]$ соответственно попарно образуют массив размерности $[1: R, 1: R O[i, 1], 1: 2]$, и в каждой из ячеек $R S[i, j, p]$ может быть записано $R 1[k, m] \mid \neg R 1[k, m]$;

в) массивы $F A S[i, j]$ и $F B S[i, j]$ имеют размерность $[1: R, 1: R O[i, 1]]$ 。 и в каждой из ячеек $R S[i, j]$ может быть записано $T 1[k, m] \mid V 1[k, m]$.

Mассив $R S$ не является АЛГОЛьным, так как записывается не число (Boolean, integer, real), а адрес, имеющий индексы.

B предлагаемом кодировании не предвидено отдельного элемента для моделирования инвертора, и запись $\neg R$ означает, что сигнал на данный вход идет или от инверсного выхода триггера, или через инвертор.

3.3. Массив выходных связей

$<$ массив выходных связей (м.вьіх.с)>::=integer array $R R::=$

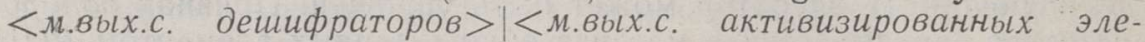
ментов>

$<$ «.вьхх.с. дешифраторов>: : = integer array $D R[1: D, 1: D O[i, 2]]$

$<$ «вых.с. активизированных элементов > : : integer array $R R[1: R, 1: R O[i, 1], 1: 3]$

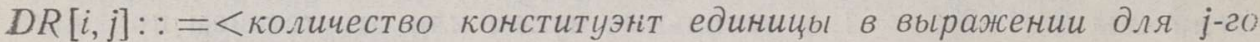
выхода дешифратора $i>$

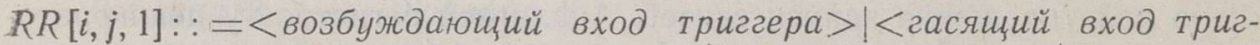
гера $\rangle \mid<$ счетный вход триггера $\rangle \mid\langle$ выход на шины $\rangle \mid\langle$ выход на задержку $\rangle \mid\langle$ прочие нагрузки $\rangle \mid\langle$ отключено $\rangle$

$R R[i, j, 2]::=[1: R]::=<$ номер регистра нагрузки $k>$

$R R[i, j, 3]::=[1: R O[k]]::=<$ номер элемента в регистре $k>$

$<$ возбуждающий вход триггера>: : =<только возбуждающий вход>|

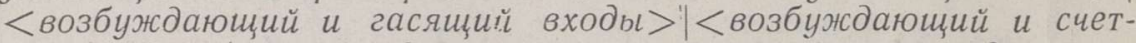

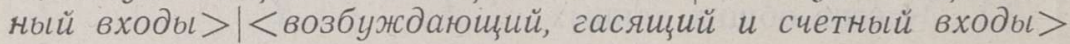

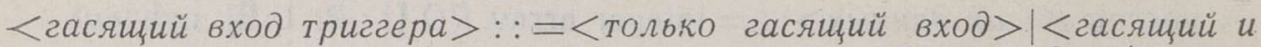

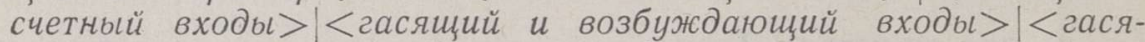
щий, счетный и возбуждающий входы $>$

$<$ счетный вход триггера $>::=\langle$ только счетный вход $\rangle \mid<$ счетный $и$ возбуждающий входы $\rangle \mid\langle$ счетный и гасящций входы $\rangle \mid\langle$ счетный, возбуждающий и гасящий входьi>

$<$ только возбуждающий вход> : : =1

$<$ только гасящий вход> : : $=2$

$<$ только сметный вход> : : $=3$

$<$ возбуждающий и гасящий входы > : : = 4

$<$ гасящий и счетный входы> : : $=5$

< счетный и возбуждающий входы > : : = 6

$<$ возбуждающцй и счетный входы> : : $=7$

<гасящий и возбуждающий входы > : : = 8

$<$ счетный и гасящий входь> : : $=9$

$<$ возбуждающий, гасящий $и$ сиетный входы> : : = 10 
<гасящий, счетный и возбуждающий входы> : : = 11

$<$ счетный, возбуждающий и гасящий входь> : : $=12$

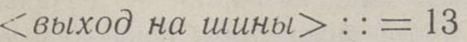

$<$ выход на задержку > : : = 14

$<$ прочие нагрузки>: : $=15$

$<$ отключено > : : $=16$

$R R[i, j]$ (для $R \neq D$ ) характеризует нагрузку, идущую за активизированным элементом.

Нагрузка типа <только...вхоо̄> (режимьь $1,2,3$ ) означает, что. в схеме не могут одновременно (т. е. в течение одного цикла моделирования) поступить сигналы на разные входы одного и того же триггера. Это соответствует условию надежной работы реальной схемы.

Нагрузка типа $\langle A u B$ входы $>$ (режимь с 4 nо 9) или типа $\langle A, B$ u $C$ входы $>$ (режимы $10,11,12$ ) (где $A, B, C$ обозначают входы триггера в произвольном порядке) означает, что выход данного активного элемента подсоединен к входу $A$ тригіера, но одновременно может поступить сигнал на $B$ и (или) $C$ входы того же триггера.

В режиме <прочие нагрузки> активизированный элемент становится эквивалентным неактивизированному, а в режиме $\langle$ отключено $\rangle$ $R 1[i, j]$ гасится после проверки режима. Режим <отключено > может применяться для исключения не нужных на данном этапе моделирования активизированных элементов без выбрасывания всего регистра й перезаписи остальных, еше нужных элементов в конец массива.

Массивом RS обладают только активизированные элементы и дешифратор.

\section{4. Массив внутренних связей}

3.4.1. Синтаксис. <массив внітренних связей> :: = <массив внутренних связей дешифраторов>

$<$ массив внутренних связей дешифраторов $>::=$ boolean array $D E[1: D$, $1: D O[i, 1], 1: D O[i, 2], 1: D R[i, k]]$

$D E[i, j, k, l]::=1 \mid 0$

3.4.2. С ем а н ти к. Одна строка по $j$ матрицы $D E$ перечисляет значения входных переменных в $l$-й конституэнте единицы в выражении для $k$-го выхода дешифратора $i$.

\section{5. Массив состояний}

3.5.1. С и н т а с и . <массив состояний $>::=<$ массив исходньх данных $>\mid<м а с с и в$ результатов $>$

$<$ массив исходнь⿻х данньх $>::=$ boolean array $R 1[1: R, 1: R O[i, 1]]$ $<$ массив результатов>: : = boolean array $R 2[1: R, 1: R O[i, 1]]$

3.5.2. С е м а н ти к а. Массив исходных данных $R 1$, свойственныг всем элементам, служит информацией для работы программы и выражает состояние схемы в моменіт начала (нового цикла) моделирования (т. е. В предшествующий момент автоматного времени). В массиве резудьтатов $R 2$ накапливается состояние схемы к концу (такта) моделирования (т. е. состояния схемы в настоящий момент автоматного времени). При переходе к новому циклу моделирования (в момент изменения автоматного времени на единицу), информация из массива $R 2$ переводится в массив R1. Массив $R 2$ при этом гасится. Массив $R 2$ имеется только у элементов памяти. 


\section{ПРИМЕРЫ КОДИРОВАНИЯ}

Пример 1

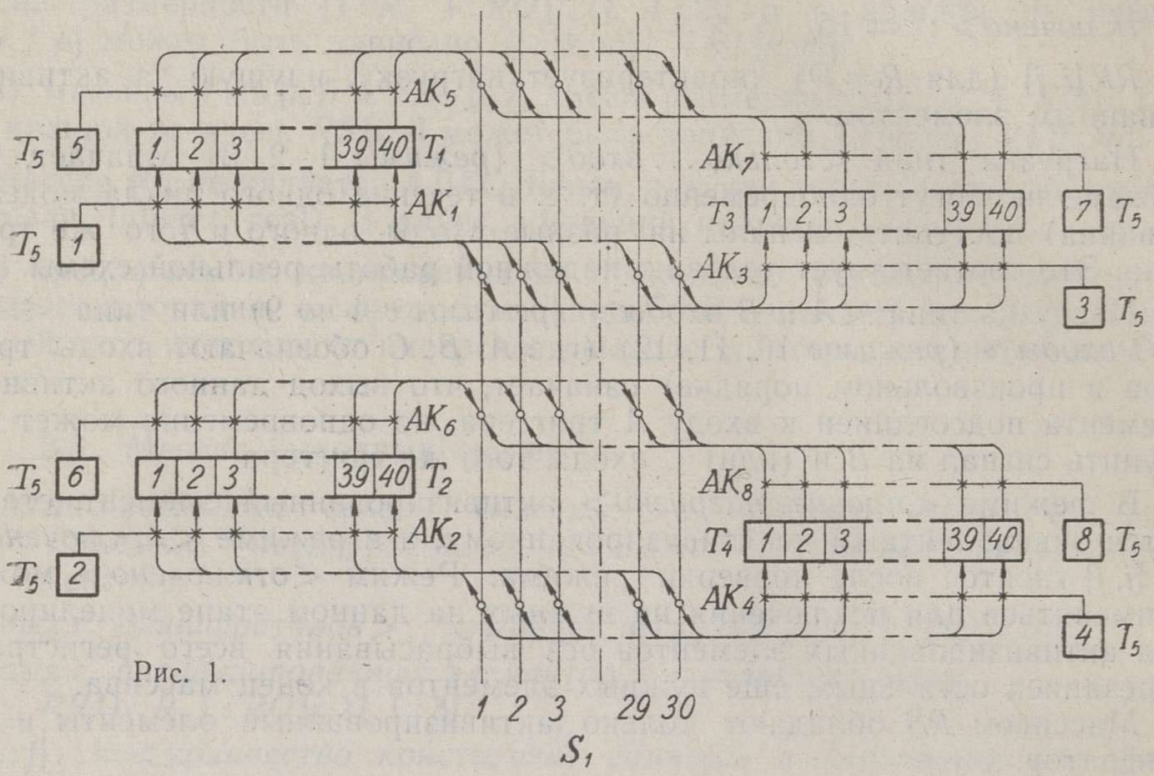

Схема на рис. 1 , работа которой состоит в пересылке информации между ретистрами через общие шины, записывает«с:

$$
\begin{aligned}
& A K=8-\text { вентили приема и передачи } \\
& T=5-4 \text { регистра хранения и } 1 \text { ре- } \\
& S=1 \\
& A=4 \text { - регистры для гашения триг- } \\
& \text { геров } \\
& I D=K=L=V=A L \\
& =A V=F A=F B=0
\end{aligned}
$$

Массивы общих данных для $A K, T$ и $A$ мөгут быть записаны АЛГОЛьными прощедурами-программами
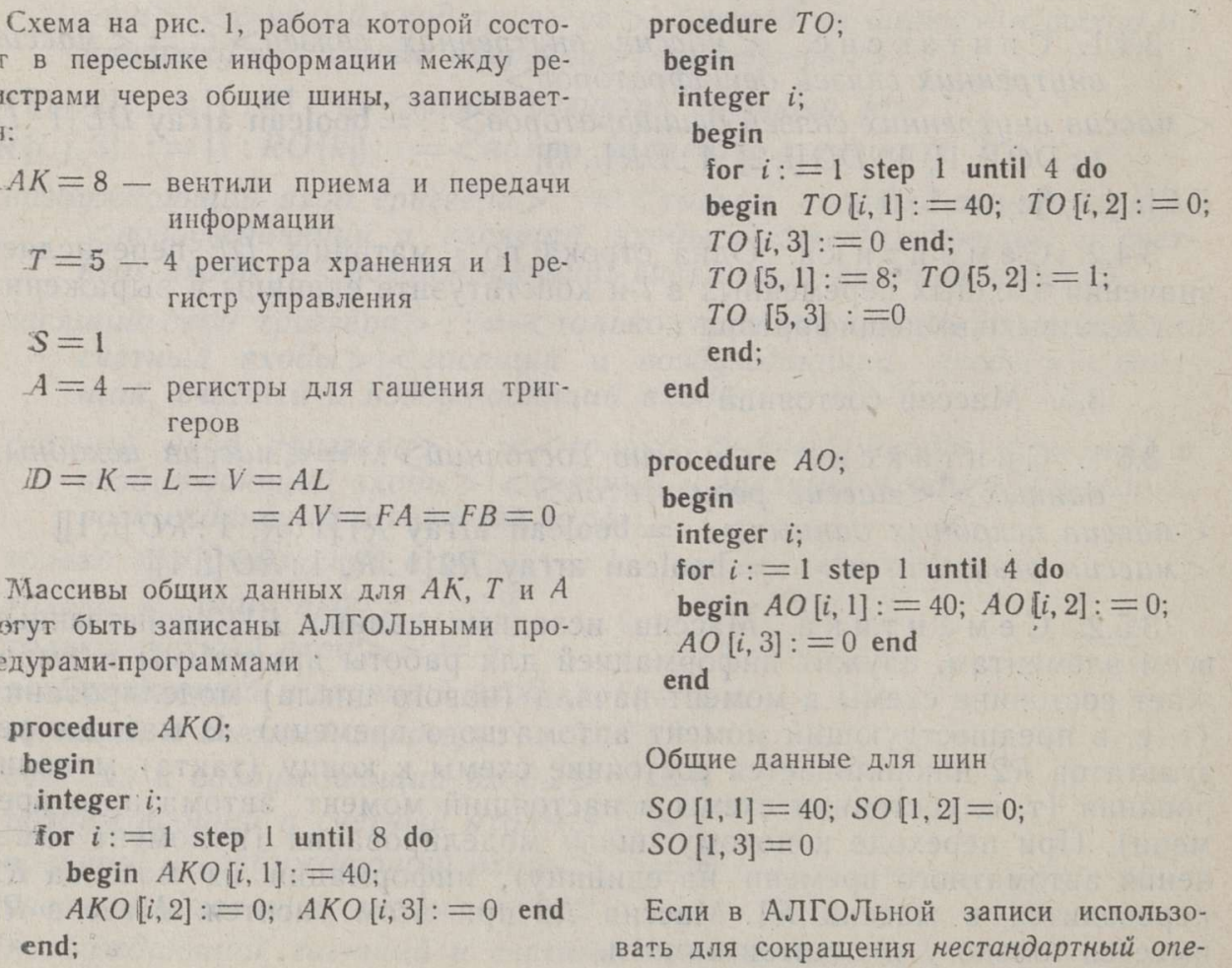

Общие данные для шин

$S O[1,1]=40 ; S O[1,2]=0$;

SO $[1,3]=0$

Если в АЛГОЛьной записи использовать для сокращения нестандартный опе- 
paтор $A \ldots B$ и понимать его как запись буквенного кода (адреса), $B$ на место (в ячейку) $A$, то массив входных связей активизированной конъюнкции будет:

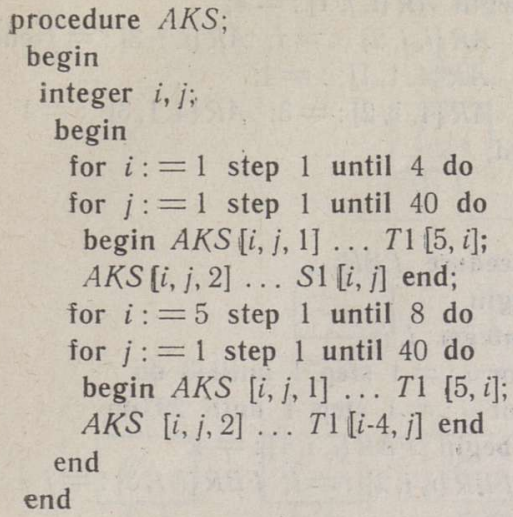

Массивы выходных связей $A K R$ и $A R$ образуются:

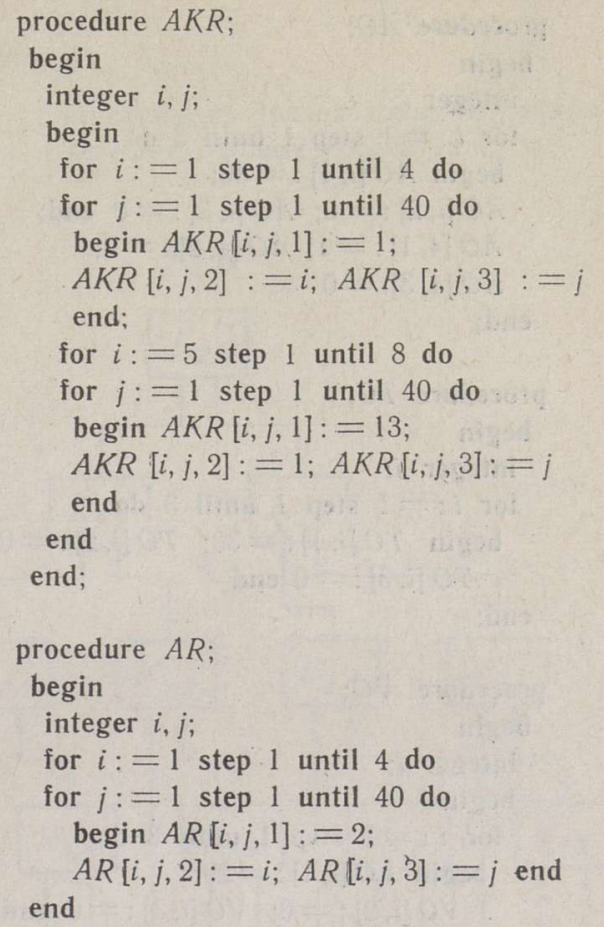

\section{Пример 2}

Последовательный сумматор, изображенный на рис. 2,

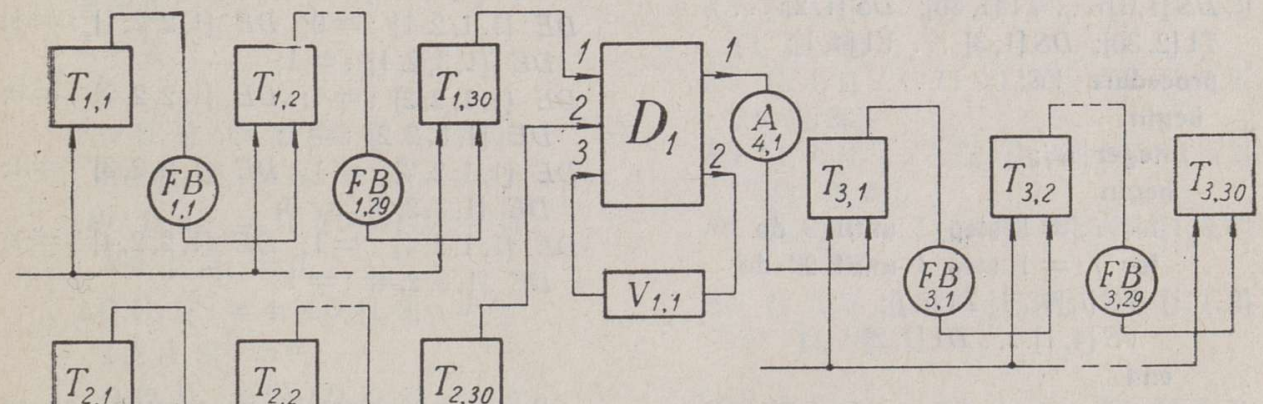

Рис. 2.

Логическая схема сумматора запишется массивами:

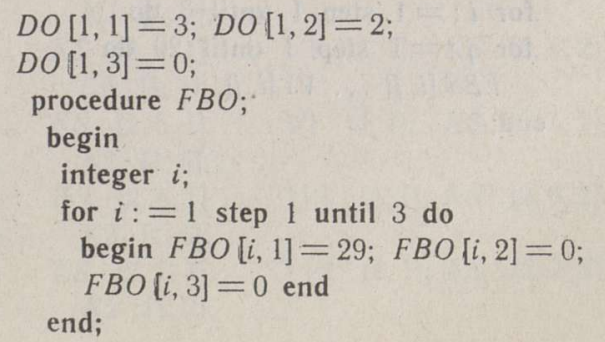




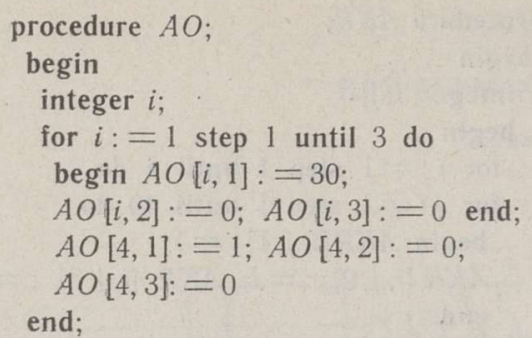

procedure $T O$;

begin

integer $i$;

for $i:=1$ step 1 until 3 do begin $T O[i, 1]:=30 ;$ TO $[i, 2]:=0$; TO $[i, 3]:=0$ end

end;
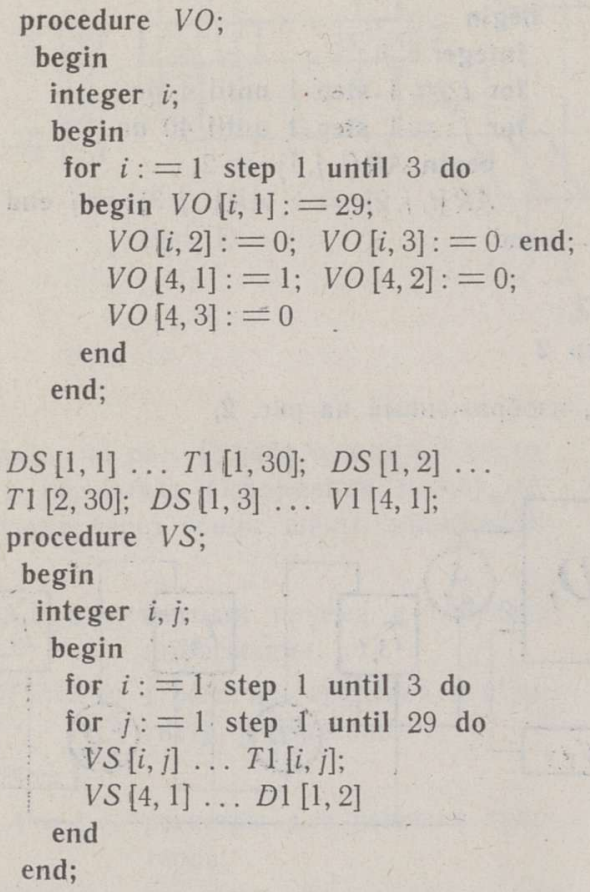

procedure $F B S$;

begin

integer $i, j$;

for $i:=1$ step 1 until 3 do

for $j:=1$ step 1 until 29 do

$F B S[i, j] \ldots V 1[i, j]$

end;

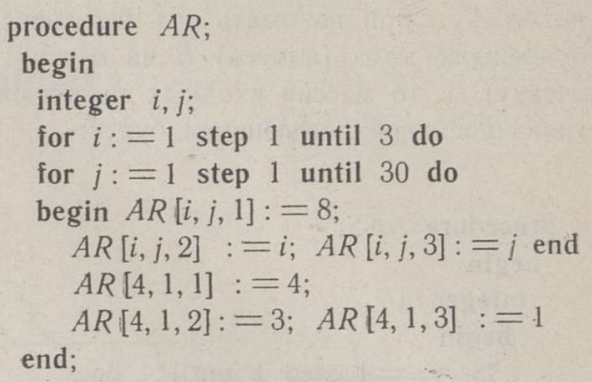

procedure $A R$;

begin

integer $i, j$;

for $i:=1$ step 1 until 3 do

for $j:=1$ step 1 until 30 do

begin $A R[i, j, 1]:=8$;

$A R[i, j, 2]:=i ; A R[i, j, 3]:=j$ end $A R[4,1,1]:=4$; $A R[4,1,2]:=3 ;$ AR $[4,1,3]:=1$ end;

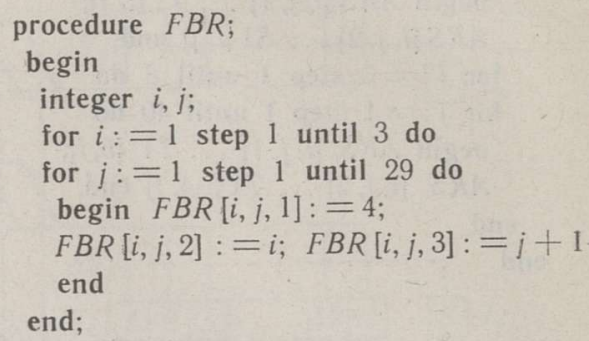

DR $[1,1]:=4$;

$D E[1,1,1,1]:=0 ; \quad D E[1,2,1,1]:=0$ : $D E[1,3,1,1]:=1$;

$D E[1,1,1,2]:=0 ; \quad D E[1,2,1,2]:=1$; $D E[1,3,1,2]:=0$

$D E[1,1,1,3]:=1 ; D E \quad[1,2,1,3]:=0$; $D E[1,3,1,3]:=0$;

$D E[1,1,1,4]:=1 ; D E[1,2,1,4]:=1$; $D E[1,3,1,4]:=1$;

$D E[1,1,2,1]:=0 ; \quad D E[1,2,2,1]:=1$; $D E[1,3,2,1]:=1$;

$D E[1,1,2,2]:=1 ; D E[1,2,2,2]:=0$; $D E[1,3,2,2]:=1$;

$D E[1,1,2,3]:=1 ; D E[1,2,2,3]:=1$; $D E[1,3,2,3]:=0$;

$D E[1,1,2,4]:=1 ; \quad D E[1,2,2,4]:=1$; $D E[1,3,2,4]:=1$

В моделирующей программе после оператора дешифратор (1) должен следовать оператор $b:=D 1[i, 1]$. 


\section{Пример 3}

В случае замены в примере 2 дешиф- тую схему из трехвходовых конъюнкций ратора на эквивалентную двухступенча- и четырехвходовых сб́орок (рис. 3 )*

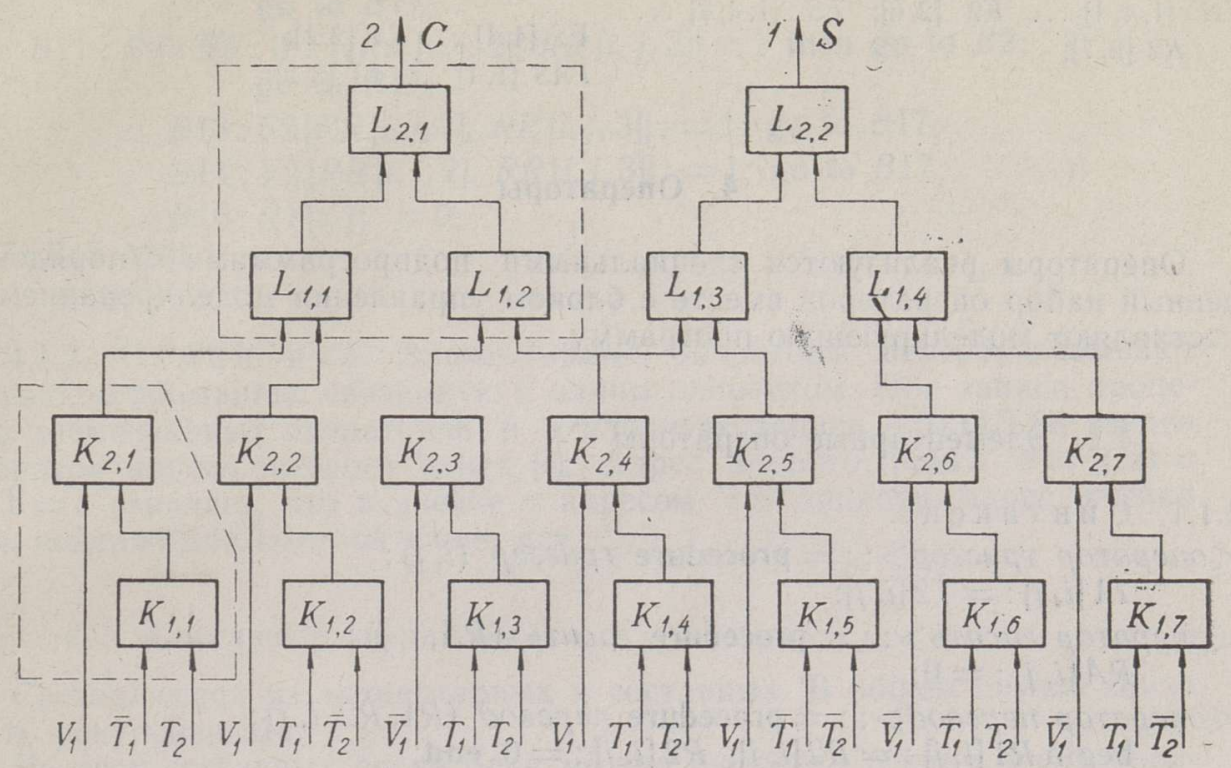

Рис. 3.

массивы для последних запишутся:

$$
\begin{aligned}
& K=2 ; \\
& L=2 ;
\end{aligned}
$$$$
K O[1,1]:=7 ; K O[1,2]:=0
$$$$
K O[1,3]:=0 \text {; }
$$$$
K O[2,1]:=7 ; K O[2,2]:=0 \text {; }
$$$$
K O[2,3]:=0 \text {; }
$$$$
\text { LO }[1,1]:=4 ; \text { LO }[1,2]:=0 \text {; }
$$$$
\text { LO }[1,3]:=0 \text {; }
$$$$
\text { LO }[2,1]:=2 ; \text { LO }[2,2]:=0 \text {; }
$$$$
\text { LO }[2,3]:=0 \text {; }
$$

$K S[1,1,1] \ldots \neg T 1 \quad[1,30] ; K S[1,1,2] \ldots$ $T 1[2,30] ;$

$K S[1,2,1] \ldots T 1 \quad[1,30] ; K S[1,2,2] \ldots$ ᄀT 12,30$]$

* На рис. 3 в обозначениях входов опущен второй индекс, так как это не вызовет неоднозначности. Пунктиром обведены одна из 3-входовых конъюнкший и одна из 4-входовых дизъюнкций.

KS $[1,3,1] \ldots T 1 \quad[1,30] ; \quad K S \quad[1,3,2] \ldots$ $T 1 \quad[2,30]$;

$K S \quad[1,4,1] \ldots T 1 \quad[1,30] ; \quad K S \quad[1,4,2] \ldots$ $T 1 \quad[2,30]$;

$K S \quad[1,5,1] \ldots \neg T 1 \quad[1,30] ; K S \quad[1,5,2] \ldots$ $\neg T 1 \quad[2,30]$;

$K S \quad[1,6,1] \ldots \neg T 1 \quad[1,30] ; K S \quad[1,6,2] \ldots$ T1 $[2,30]$;

$K S \quad[1,7,1] \ldots T 1 \quad[1,30] ; K S \quad K 1,7,2] \ldots$ $\neg T 1 \quad[2,30]$;

$K S \quad[2,1,1] \ldots$ V1 $[4,1] ; \quad K S \quad[2,1,2]$. $K 2 \quad[1,1]$;

$K S \quad[2,2,1] \ldots V 1 \quad[4,1] ; \quad K S \quad[2,2,2] \ldots$ K2 $[1,2]$;

KS $[2,3,1] \ldots 7 V 1 \quad[4,1] ; K S[2,3,2] \ldots$ K2 $[1,3]$;

$K S \quad[2,4,1] \ldots V 1 \quad[4,1] ; \quad K S \quad[2,4,2] \ldots$ $K 2 \quad[1,4]$;

$K S \quad[2,5,1] \ldots V 1 \quad[4,1] ; \quad K S \quad[2,5,2] \ldots$ K2 $[1,5]$;

$K S \quad[2,6,1] \ldots \neg V 1 \quad[4,1] ; K S \quad[2,6,2] \ldots$ $K 2 \quad[1,6]$;

$K S \quad[2,7,1] \ldots \neg V 1 \quad[4,1] ; K S \quad[2,7,2] \ldots$ K2 $[1,7]$; 


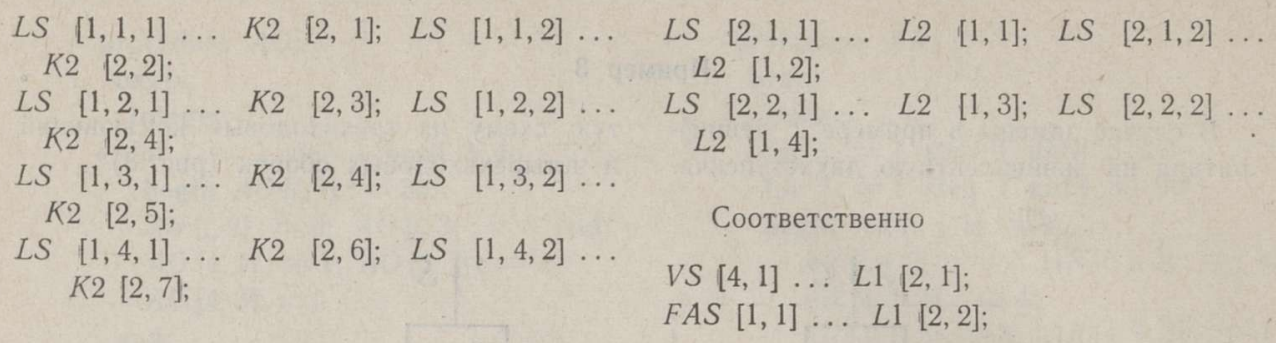

\section{4. Операторы}

Операторы реализуются специальными подпрограммами. Упорядоченный набор операторов вместе с блоком управления моделированием сеставляют моделирующую программу.

\section{1. Элементарные операторы}

4.1.1. С ин т а к и и с.

$<$ оператор тригге $>::=$ procedure триггер $(i, j)$;

$T 1[i, j]:=T 2[i, j]$

$<$ оператор гасить $>::=$ procedure гасить $(R A, i, j)$;

$R A[i, j]:=0$;

$<$ оператор перевод>: : = procedure перевод $(R 1, R 2, i, j)$;

begin $R 1[i, j]:=R 2[i, j] ; R 2[i, j]:=0$ end;

<оператор сборка>:: = procedure сборка $(i, j)$;

$L 1[i, j]:={ }^{2} L S[i, j, 1] \vee^{2} L S[i, j, 2]$

<оператор конъюнкция>: : = procedure конъюнкция $(i, j)$;

$K 1[i, j]:={ }^{2} K S[i, j, 1] \wedge{ }^{2} K S[i, j, 2] ;$

$<$ оператор дешифратор >: : = procedure дешифратор (i);

begin

for $i:=1$ step 1 until $D O[i, 1]$ do

$D 3[j]:={ }^{2} D S[i, j]$

$B 1:$ for $k:=1$ step 1 until $D O[i, 2]$ do

$B 2:$ for $l:=1$ step 1 until $D R[i, k]$ do

begin

integer $j$;

$D 1[i, k]:=0$;

$B 3:$ for $j:=1$ step 1 until $D O[i, 1]$ do

if $D E[i, j, k, l]=D 3[j]$ then go to $B 3$

else go to $B 2$;

$D 1[i, k]:=1$; go to $B 1$

end

end;

$<$ оператор задержка>: : = procedure задержка $(i, j)$;

$V 2[i, j]:={ }^{2} V S[i, j]$

<оператор выход>: := procedure выход $(R R, i, j)$;

begin

switch $u 1[R R[i, j, 1]]:=B 1, B 2, B 3, \ldots, B 14, B 15, B 16$;

begin

if $b=1$ then go to $u 1[R R[i, j, 1]]$; go to $B 17$; 
$B 1: T 2[R R[i, j, 2], R R[i, j, 3]]:=1$; go to $B 17$;

$B 2: T 2[R R[i, j, 2], R R[i, j, 3]]:=0$; go to $B 17$;

$B 12: B 9: B 6: B 3: T 2[R R[i, j, 2], R R[i, j, 3]]:=\neg T 2[R R[i, j, 2]$, $R R[i, j, 3]$; go to $B 17$;

$B 10: B 7: B 4:$ if $T 1[R R[i, j, 2], R R[i, j, 3]]=0$ then go to $B 1$; go to $B 17$;

$B 11: B 8: B 5:$ if $T 1[R R[i, j, 2], R R[i, j, 3]]=1$ then go to $B 2$; go to $B 17$;

$B 13: S 2[R R[i, j, 2], R R[i, j, 3]]:=1$; go to $B 17$;

$B 14: \operatorname{V2}[R R[i, j, 2], R R[i, j, 3]]:=1$; go to $B 17$;

$B 17: B 15:$ end

$B 16: R 1[i, j]:=0$;

end;

4.1.2. С ем антика. Элементарные операторы переобразовывают только информацию, связанную с одним элементом. При записи процедур элементарных операторов в эталонную запись АЛГОЛ-60 введен оператор адреса второго ранга $\left[{ }^{3}\right]$. Адрес второго ранга ${ }^{2} a=^{\prime}\left({ }^{\prime} a\right)=$ $={ }^{\prime} \mathrm{b}=\mathrm{c}$ означает, что в ячейке с адресом «а» записан адрес ячейки «b», содержимое которой равно «с».

\section{2. Составные операторы}

Составляются из элементарных и составных. В общем случае могут быть рекурсивными.

Из всех составных операторов можно выделить группу оператороврегистров, реализующих регистры схемы. В данном предварительном гарианте кодирования другие группы не выделены.

4.2.1. Операторы - регистры.

<оператор триггерный регистр>::= procedure триггерный регистр (i); begin

integer $j$;

for $j:=1$ step 1 until $T O[i, 1]$ do

end; тригеер $(i, j)$

$<$ оператор регистр сборок>::= procedure регистр сборок (i);

begin

integer $j$;

own array $L S[1: L, 1: L O[i, 1], 1: 2]$;

for $j:=1$ step 1 until $L O[i, 1]$ do

сборка $(i, j)$

end;

$<$ оператор дешифраторы >:: = procedure дешифраторы;

begin

integer $i, j, k, l$;

own array $D S[1: D, 1: D O[i, 1]]$;

own integer array $D R[1: D, 1: D O[i, 2]]$;

own boolean array $D E[1: D, 1: D O[i, 1], 1: D O[i, 2], 1: D R[i, k]] ;$

for $i:=1$ step 1 until $D$ do

end: деширратор $(i)$ 
$<$ оператор регистр задержек>::=procedure регистр задержек $(i)$;

begin

integer $j$;

own array $\operatorname{VS}[1: V, 1: \operatorname{VO}[i, 1]]$;

for $j:=1$ step 1 until $V O[i, 1]$ do

end; задержка $(i, j)$

$<$ оператор регистр конъюнкции>:: = procedure регистр конгюнкции (i); begin

integer $b, j, m$;

own array $K S[1: K, 1: K O[i, 1], 1: 2]$;

switch $w 1[\mathrm{~m}]:=Q 3, Q 4, Q 4$;

switch $w 2[m]:=Q, Q 1, Q 2$;

comment : метка $Q 1$ является переходом к следующему (т. е. $i+1)$ регистру;

Q2: for $j:=1$ step 1 until $K O[i, 1]$ do

begin

if $j=1$ then $m:=1$; go to $\operatorname{wl}[\mathrm{m}]$;

Q3: if $K O[i, 2]=0$ then $m:=2$ else $m:=3$;

if $K S[i, j, 1]=0$ then go to $w 2[m]$;

go to $w l[\mathrm{~m}]$;

Q4: конъюнкция $(i, j)$

end; end

$<$ оператор активизированный регистр >:: = procedure активизированный регистр (элемент, R, RO, RS, R1, RR, i);

begin

integer $j$;

own array $R S[1: R, 1: R O[i, 1]]$

own integer array $R R[1: R, 1: R O[i, 1], 1: 3]$;

for $j:=1$ step 1 until $R O[i, 1]$ do

end:

активизированный элемент (элемент, $R 1, R R, i, j$ )

$<$ оператор гашение регистра>::= procedure гашение регистра (RA, $R O, i)$;

begin

integer $j$;

for $j:=1$ step 1 until $R O$ do

end; гасить $(R A, i, j)$

$<$ оператор перевод регистра>::= procedure перевод регистра $(R O$, $R 1, R 2, i)$;

begin

integer $j$;

for $j:=1$ step 1 until $R O$ do

end;

перевод $(R 1, R 2, i, j)$ 


\subsection{2. Прочие операторы.}

<оператор активизированный элемент >: : = procedure активизированный элемент (элемент, $R 1, R R, i, j$ );

begin

integer $b$;

begin

элемент:

$b:=R 1[i, j]$

end BulxO2 $(R R, i, j)$

end;

$<$ оператор схема >: : = procedure $с$ хема $($ регистр, $R)$;

begin

integer $i, R$;

$Q 1$ : for $i:=1$ step 1 until $R$ do

end; регистр $(i)$

$<$ оператор гашение $>::=$ procedure гашение $(R A, R, R O)$;

begin

integer $R, R O, i$;

for $i:=1$ step 1 until $R$ do

гашение регистра $(R A, R O, i)$

end;

<оператор время>: : = procedure время $(R, R O, R 1, R 2)$;

begin

integer $R, R O, i$;

for $i:=1$ step 1 until $R$ do

end; перевод регистра $(R O, R 1, R 2, i)$

<оператор счетчик>: : = procedure счетчик $(i)$;

$$
Z[i]:=Z[i]+1 \text {; }
$$

$<$ оператор гашение счетчиков $>:$ : = procedure гашение счетчиков $(Z O)$; begin

integer $Z O, i$;

for $i:=1$ step 1 until $Z O$ do

end;

$Z[i]:=0$

<оператор начало>: : = procedure начало;

begin

гашение счетииков ( $Z O)$;

гашение $(D 1[i, j], D, D O[i, 1])$;

гашение ( $K 1[i, j], K, K O[i, 1])$;

гашение ( $L 1[i, j], L, L O[i, 1])$;

гашение ( $S 1[i, j], S, S O[i, 1])$;

гашение $(T 2[i, j], T, T O[i, 1])$; 


\section{гашение (V2[i,j], $V, V O[i, 1])$; \\ гашение (AK1[i,j], $A K, A K O[i, 1])$; \\ гашение (AL1 $[i, j], A L, A L O[i, 1])$; \\ гашение (AV2 $[i, j], A V, A V O[i, 1])$; \\ гашение (FA1[i,j], FA, FAO $[i, 1])$; \\ гашение (FB1 $[i, j], F B, F B O[i, 1])$}

end;

\section{Пример 4}

Для моделирования схемы на рис. 1 программа может иметь вид:

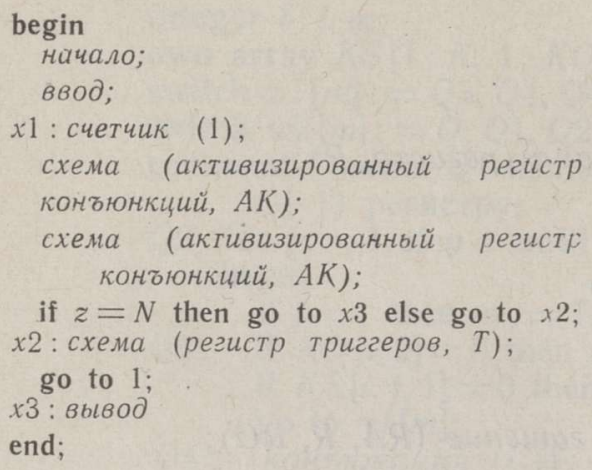

\section{ЛИТЕ РА Т У Р А}

Управление моделированием легче всего осуществить в месте, где поставлена проверка счетчика, а также при возвращении к метке $x 1$.

В программе использованы не описанные в статье операторы ввод и вывол. Их содержание зависит от примененного конкретного АЛГОЛьного транслятора.

Использование дважды подряд процедуры схема (активизированный регистр конъюнкций, $A K)$ вызвано наличием в схеме шин.

1. Глушко в В. М., Синтез цифровых автоматов, Физматиздат, М.-Л., 1962.

2. Бэ к у с Дж. В. и др., Ж. вычислит. матем. и. математич. физики, 1, № 2, 308-342 (1961).

3. Г л уш к о В В. М., Ю ще н к о Е. Л., Вычислительная машина «Қиев», ГИТЛ УССР, Киев, 1962.

4. Б о т те н б р ух Г., Структура АЛГОЛ-60 и его использование, Изд. ИЛ, М., 1963.

Институт кибернетики

Академии наук Эстонской ССР
Поступила в редакцию

31 I 1964

\section{KODEERING NUMBRILISTE SKEEMIDE KIRJELDAMISEKS JA MODELLEERIMISEKS}

\section{H. Salum}

\section{Resümee}

Esitatakse kodeeringu algvariant. Modelleeritav skeem peab olema esitatud loogilise struktuurskeemina registrite ja elementide nivool. Kodeerimise kirjeldamisel kasutatakse ALGOL-60 [ $\left.{ }^{2,4}\right]$ sümboleid. Peale selle kasutatakse teise järgu aadressi mōistet [3]. Tuuakse näiteid konkreetsete skeemide kirjeldamise kohta.

\section{Eesti NSV Teaduste Akadeemia Küberneetika Instituut}

Saabus toimetusse 31. I 1964

\section{A CODING FOR DESCRIBING AND SIMULATING DIGITAL SCHEMES}

\section{H. Salum}

\section{Summary}

A preliminary variant of coding is given. A scheme to be described and simulated has to be presented as a composition ['] of logical elements and registers. The ALGOL-60 symbols are used to describe the coding. An operator of the second-range-address $\left.{ }^{3}\right]$ is used to simplify the descriptions. Some examples of the applications are presented.

Academy of Sciences of the Estonian S.S.R.,

Received Institute of Cybernetics 